\title{
Microstructure and the Pulsar Emission Mechanism
}

\author{
Valentín Boriakoff \\ National Research Council, US; Phillips Lab, Hanscom AFB, MA, US
}

\section{Observational Facts}

Pulsar radio emission is composed in varying proportions of microstructure, subpulses and amorphous emission. Each component has its own time and frequency behaviour. The average pulse profile changes its width with frequency, typically as $\nu^{-0.26}$. The location (pulse phase) of the subpulses refered to a fiducial point within the profile follows the same $\nu^{-0.26}$ law as the width of the average pulse profile. The fiducial point in the pulse profile follows the interstellar plasma dispersion relation exactly: the source emission at this phase is simultaneous at all frequencies. The subpulse width is typically nearly constant with frequency. Emission of a subpulse may be systematically absent at certain frequency ranges, the pulse profile may be different there. Micropulses are typically very wideband: $\sim 1 \mathrm{GHz}$ or more. Individual micropulse widths are constant with frequency. Its time width distributions have upper limits $(0.1-1 \mathrm{~ms})$. The time separation (phase) of any micropulse with respect to the fiducial point is constant with frequency, i.e. it is simultaneous at all frequencies at source. The spectrum of individual micropulses appears to have breakpoints: it is observable at $\nu \leq \nu_{(\mu c o)}$, but not above it. This effect (and not a steep spectrum) seems to account for the smaller number of micropulses at higher frequencies: at $\nu$ we see micropulses with $\nu_{(\mu c o)}>\nu$ only, micropulses with $\nu_{\left(\mu c_{0}\right)}<\nu$ are not visible, at low frequencies we will see more micropulses than at high frequencies. Micropulses prefer the conal emission zones, and are very weak or absent in the core emission zones. More than one micropulse can be observed at a given time. Micropulses occasionally appear in trains with a nearly constant time separation, up to 20 micropulses have been observed in such quasi-periodic sequences. Sequence phase and period are variable within a train, they may have discrete values. Such trains can be observed simultaneously at widely separated frequencies. Ocasionally more than one quasi-periodic train may appear at the same time. Subpulses in general follow the linear polarization angle of the average pulse profile, with occasional $90^{\circ}$ jumps. Within a micropulse the polarization angle can be constant, or can have a fast swing of $\sim 50^{\circ}$. Micropulse polarization angle can be widely different from that of the subpulse on which it is superimposed.

\section{Interpretation and Conclusions}

I will use Buschauer \& Benford's $(1976,1980)$ coherent emission model. I assume that the radius-to-frequency mapping operates, and that radiation is peaked in the direction tangential to the local magnetic field line. I propose: 
1) Micropulses and subpulses are generated in approximately the same volume of the pulsar magnetosphere, because they often appear associated. Single appearances of both comprise many elementary radiators (bunches or bunch organizations) radiating independently. The mechanism of emission of micropulses and subpulses is the same, but it is different in the coherence length: long lengths (several bunches) produce narrowband emission that form subpulses, short lengths (single bunch) produce wideband emission that form micropulses. 2) This explains the simultaneous emission at source for all the frequencies of the micropulse, since the basic emission is by a single bunch that is wideband and simultaneous at all frequencies. Micropulses have spectra similar to that of the short coherence length mechanism, with a cutoff frequency apparent. Micropulses have to be generated within a narrow flux tube, it will confine the many emitting single bunches to this small cross-section where the emission peaked on tangents to the magnetic field lines point towards us. The width of the rnicropulse is given by the width of the confining small flux tube. The radiusto-frequency mapping will determine the visibility of the micropulse generated at that height, other micropulses where $\nu_{(\mu c o)}>\nu$ and the tangent is pointing towards us will be visible. This is why there are less micropulses at higher frequencies, we see at $\nu$ micropulses with $\nu_{(\mu c o)} \geq \nu$ only.

3) The subpulses must be made of many different narrowband mechanisms, each operating at a narrow frequency range, to explain the divergent pulse-phase location at lower frequencies. By placing the narrowband emitting mechanisms at different heights from the neutron star the subpulse phase location and widening of the pulsar profile for lower frequencies are accounted for. The absence of emission at some frequencies is explained by the emission mechanism not operating at those heights, it leaves the subpulse emission at other heights uniaffected. Physical association frequently observed between subpulses at different frequencies (at different phases) is explained by the confinement of the emitting regions at different heights by a section of the magnetic field where radioemission can be generated, a flux tube. It should be sufficiently wide in angle to be able to have emission over typical subpulse time widths. Many particle bunch trains radiating coherently and located close to a specific height will be seen simultaneously in a single subpulse at a given frequency.

4) Polarization behaviour differences between micropulses and subpulses are explained by the difference in location between the emitting regions. For micropulses the polarization angle swings can be explained by the extent of the small flux tube.

5) Quasi-periodicities in the microstructure are explained by a mechanism that spaces the location of the small flux tubes, or a temporal modulation mechanism, for example vibrations of the neutron star.

By assuming that both short and long coherence lengths are present in the magnetosphere of a single pulsar we are able to explain many of the known characteristics of the micropulse and subpulse emission.

\section{References}

Buschauer, R., \& Benford, G., 1976, MNRAS, 177, 109

Buschauer, R., \& Benford, G., 1980, MNRAS, 190, 945 